\title{
Aortic valve replacement in young patients: should the biological prosthesis be recommended over the mechanical?
}

\author{
Alberto Alperi, Daniel Hernandez-Vaquero, Isaac Pascual, Rocio Diaz, Iria Silva, Ruben Alvarez-Cabo, \\ Pablo Avanzas, Cesar Moris \\ Heart Area, Hospital Universitario Central de Asturias, Oviedo, Spain \\ Correspondence to: Daniel Hernandez-Vaquero, MD, PhD. Heart Area, Hospital Universitario Central de Asturias, Oviedo, Spain. \\ Email: dhvaquero@gmail.com. \\ Provenance: This is a Guest Editorial commissioned by Section Editor Busheng Zhang, PhD, MD (Department of Cardiac Surgery, Shanghai Chest \\ Hospital, Shanghai Jiaotong University, Shanghai, China). \\ Comment on: Schnittman SR, Adams DH, Itagaki S, et al. Bioprosthetic aortic valve replacement: Revisiting prosthesis choice in patients younger than \\ 50 years old. J Thorac Cardiovasc Surg 2018;155:539-47.e9.
}

Submitted Jan 22, 2018. Accepted for publication Feb 08, 2018.

doi: 10.21037/atm.2018.02.21

View this article at: http://dx.doi.org/10.21037/atm.2018.02.21

Schnittman and colleagues have recently published a very interesting observational study from the Mount Sinai group (1). It contributes with new and useful data on the controversial issue of the most adequate aortic valve type when performing an aortic valve replacement (AVR) in young patients (under 50 years old). The results of this study are reinforced by the important number of patients analysed, which was higher than in most of the clinical trials performed about this topic previously (2-4). They concluded that 15 -year mortality in patients younger than 50 years of age who underwent an AVR using biological aortic valves is similar (around $80 \%$ ) than those using mechanical prosthesis. Less strokes and bleeding complications were observed in the bioprosthetic group, although the rate of reoperations in those patients was markedly higher $(24.6 \%$ vs. $8.1 \%$ in the mechanical group). Even though, there are some questions that may raise concern.

First of all, authors (1) admitted that data were collected from administrative database by non-clinical staff. In addition, a lack of information regarding important issues such as prosthesis model or size is observed. There is also no information about left ventricle ejection fraction (LVEF) during the follow-up. LVEF was demonstrated to be a strong and independent predictor of mortality after AVR $(5,6)$. Therefore, we think that an equal distribution of this factor between groups would be important.

The cohort of patients included in the final analyses was restricted. Patients with concomitant coronary artery bypass or congenital heart disease were excluded, as those with previous or concomitant valve replacement or repair of any other valve. Young patients with rheumatic aortic valve disease would represent a numerous group of AVR in less than 50-year-old patients, especially within developing countries, but lot of them would not be included in this study due to concomitant mitral or tricuspid disease needing intervention.

Twenty percent of patients in each group underwent concomitant ascending aortic surgery, but there is no specification on which diseases they had and on which procedures were performed. This would be important as mortality and complication rates are different depending on it.

Patients with mechanical heart valves were at an increased risk of stroke compared with patients without, and they require continuous anticoagulation. But there was no information about the international normalised ratio during the follow-up for this oral anticoagulation therapy (OAC). This should be taken into account for a better interpretation of the stroke and major bleeding rates.

Chronic OAC plays an important role in patient's decision. Even when permanent OAC is necessary after biological valve implantation (patients with atrial fibrillation, previous pulmonary embolism and so on), there are new direct OAC agents (DOAC) with more reliable pharmacodynamics and have more effective and safety 
profiles. Although strong evidence is lacking, these new DOAC therapies have shown good results within patients with biological valves and could therefore be used in this setting $(7,8)$. Conversely, they are not safe and therefore, not recommended after mechanical valve implantation (9).

Reoperation procedures are challenging interventions with higher risk of mortality. The 30-day mortality rate of $4.8 \%$ observed in this study is a great result, which could not be achieved in other less experienced surgical groups. Internal results of the surgical team should be emphasized when explaining younger patients about their choices before surgery.

Life expectancy in developed countries is currently above 80 years old. When evaluating patients with a median age of 43 years who undergo AVR, their life expectancy can be similar than that of the normal age-related population with no medical conditions. Although authors have reported a median follow-up of 11.8 years, this may be even insufficient in this clinical setting. Thus, longer follow-up periods of 30 and 40 years would be extremely important to increase knowledge.

This study allows us to understand more deeply the natural history of patients with mechanical and biological prosthesis. These patients had similar mortality, approximately $20 \%$ at 15 years follow-up. Those with biological valve had less stroke and bleeding complications. Conversely, less reintervention rates were observed with mechanical prosthesis, but this difference is only noticed after 7 years of follow-up, rising 5 times higher at 15 years.

We are witnessing an exponential increase in biological prostheses for different reasons. The valve in valve procedure is emerging as a useful technique in this clinical context (9). Nevertheless, biological prostheses cannot be recommended as a general rule for patients under 60 years old until future studies with longer follow-up period show that biological prosthesis are as safe as the mechanical prostheses.

\section{Acknowledgements}

None.

Cite this article as: Alperi A, Hernandez-Vaquero D, Pascual I, Diaz R, Silva I, Alvarez-Cabo R, Avanzas P, Moris C. Aortic valve replacement in young patients: should the biological prosthesis be recommended over the mechanical? Ann Transl Med 2018;6(10):183. doi: 10.21037/atm.2018.02.21

\section{Footnote}

Conflicts of Interest: The authors have no conflicts of interest to declare.

\section{References}

1. Schnittman SR, Adams DH, Itagaki S, et al. Bioprosthetic aortic valve replacement: Revisiting prosthesis choice in patients younger than 50 years old. J Thorac Cardiovasc Surg 2018;155:539-47.e9.

2. Hammermeister K, Sethi GK, Henderson WG, et al. Outcomes 15 years after valve replacement with a mechanical versus a bioprosthetic valve: final report of the Veterans Affairs randomized trial. J Am Coll Cardiol 2000;36:1152-8.

3. Stassano P, Di Tommaso L, Monaco M, et al. Aortic valve replacement: a prospective randomized evaluation of mechanical versus biological valves in patients ages 55 to 70 years. J Am Coll Cardiol 2009;54:1862-8.

4. Oxenham H, Bloomfield P, Wheatley DJ, et al. Twenty year comparison of a Bjork-Shiley mechanical heart valve with porcine bioprostheses. Heart 2003;89:715-21.

5. Tjang YS, van Hees Y, Körfer R, et al. Predictors of mortality after aortic valve replacement. Eur J Cardiothorac Surg 2007;32:469-74.

6. Vassiliou V, Chin C, Perperoglou A, et al. Ejection Fraction by Cardiovascular Magnetic Resonance Predicts Adverse Outcomes Post Aortic Valve Replacement. Heart 2014;100:A53-4.

7. Di Biase L. Use of Direct Oral Anticoagulants in Patients With Atrial Fibrillation and Valvular Heart Lesions. J Am Heart Assoc 2016;5.

8. Nardi F, Gulizia MM, Colivicchi F, et al. ANMCO Position Paper: direct oral anticoagulants for stroke prevention in atrial fibrillation: clinical scenarios and future perspectives. Eur Heart J Suppl 2017;19:D70-88.

9. Dvir D, Webb JG, Bleiziffer S, et al. Transcatheter aortic valve implantation in failed bioprosthetic surgical valves. JAMA 2014;312:162-70. 